\title{
Automatic Speech Segmentation Based on Acoustical Clustering*
}

\author{
Jon A. Gómez, Emilio Sanchis, and María J. Castro-Bleda \\ Departamento de Sistemas Informáticos y Computación, \\ Universidad Politécnica de Valencia, Spain \\ \{jon, esanchis, mcastro\}@dsic.upv.es \\ http://elirf.dsic.upv.es
}

\begin{abstract}
In this paper, we present an automatic speech segmentation system based on acoustical clustering plus dynamic time warping. Our system operates at three stages, the first one obtains a coarse segmentation as a starting point to the second one. The second stage fixes phoneme boundaries in an iterative process of progressive refinement. The third stage makes a finer adjustment by considering some acoustic parameters estimated at a higher subsampling rate around the boundary to be adjusted. No manually segmented utterances are used in any stage.

The results presented here demonstrate a good learning capability of the system, which only uses the phonetic transcription of each utterance. Our approach obtains similar results than the ones reported by previous related works on TIMIT database.
\end{abstract}

Keywords: automatic speech segmentation, phoneme boundaries detection, phoneme alignment.

\section{Introduction}

It is well known the usefulness of phonetically segmented speech corpora for several purposes. Lately, there is a special attention in the selection of phonetic units for Text-To-Speech (TTS) systems. However, the availability of segmented speech databases for training acoustic models continues being of interest in the construction of Automatic Speech Recognition (ASR) systems.

The manual segmentation of speech corpora is a hard work which implies many hours of human phonetic experts, and it does not avoid some deviations due to different human expert criteria. Some researchers have given the same speech database to different human experts to segment it. Then, they evaluated the difference between the manual segmentations obtained. In [1], 97\% of the boundaries within a tolerance interval of $20 \mathrm{~ms}$ were found, and $93 \%$ in [2].

Our method does not need any subset of manually segmented sentences for bootstrapping. The input to our system are both the speech signal and the known phonetic sequence of each utterance. It fixes correctly $88 \%$ of boundaries

^ Work supported by the Spanish MICINN under contract TIN2008-06856-C05-02.

E.R. Hancock et al. (Eds.): SSPR \& SPR 2010, LNCS 6218, pp. 540-548, 2010.

(C) Springer-Verlag Berlin Heidelberg 2010 
within a $20 \mathrm{~ms}$ tolerance interval compared to a manual segmentation. Therefore, their output is suitable for training acoustic models in ASR systems, such as those based on Hidden Markov Models (HMM), Neural Networks or hybrid systems. This method was used on selecting phonetic units for TTS as part of a comparative study. It obtained better results than HMM in an objective test, but worse in a perceptual one [3].

A work for speech/music discrimination of radio recordings that uses similar techniques was presented in [4]. Their goal is to segment audio streams by classifying each segment as either speech or music, while our goal is the adjustment of phonetic boundaries. Both our and their system operate on three stages and use dynamic programming for optimal segmentation. Nevertheless, their dynamic programming algorithm discriminates speech against music using posterior class probabilities estimated by means of Bayesian Networks. Our dynamic time warping algorithm uses posterior phonetic probabilities computed as described below in Section 2.2.

Next section describes our automatic segmentation system. Section 3 explains the measures used for evaluating segmentation accuracy. Section 4 presents experimental results and discuses them, and Section 5 concludes.

\section{The Speech Segmentation System}

Our automatic speech segmentation system attempts to solve the problem in three stages. The first stage estimates a coarse segmentation which is used as a starting point to the second one. The second stage does a progressive refinement of phoneme boundaries by means of a dynamic time warping (DTW) algorithm which uses phonetic probabilities estimated at each frame. The third stage adjusts the boundaries in a more precise manner.

A previous version of our system was presented [5] and it worked in two stages. The coarse segmentation module fixed phoneme boundaries following a knowledge-based approach by using a set of language-dependent acousticphonetic rules. New coarse segmentation is language-independent and positions phoneme boundaries based on statistical analysis of acoustic parameters. The whole system has been adapted to be language-independent. Results for both English and Spanish languages are presented in Section 4.

\subsection{Coarse Segmentation}

Initial phoneme boundaries are positioned using classification techniques at different levels and doing four consecutive steps. First and second steps are applied to each sentence individually. In the first step, time marks are placed where acoustic changes are considered relevant. Every two consecutive time marks define an acoustic segment. In the second step, acoustic segments are associated with phonetic units following simple acoustic-phonetic rules. In the third step, the previous association is used to estimate a Gaussian Mixture Model (GMM) with several Gaussians per phonetic unit. A new association of acoustic segments 
with phonetic units is performed based on phonetic probabilities from the GMM, then, an iterative process refines the GMM until no changes in association are found. In the fourth step, time marks from step 1 are not used as reference, so phoneme boundaries are fixed by using only the phonetic probabilities. The first iteration of this step uses the phonetic probabilities provided by the GMM computed in the last iteration of previous step. The iteration stops when no changes on boundary positions are found. The algorithm follows:

1) Location of time marks in relevant acoustic changes. Time marks are placed between two consecutive frames which are classified in different classes. This strategy gives us a sequence of time marks where a phoneme boundary is located with high probability. Here, time marks are established in three levels. The first level does clustering with two Gaussian distributions using two parameters: energy $(E)$ and the first cepstral coefficient $(C C 1)$. Then, frames are classified into one of the two classes, and a time mark is fixed when frame class changes. The next level performs clustering with three Gaussian distributions, using again $E$ and $C C 1$, and new time marks are obtained, most of them on the same location than the previous marks. With these clustering processes, boundaries between fricatives and no fricatives are found by the first level, the second level confirms them and find new boundaries between silence and no silence. The third clustering process works inside the acoustic segments delimited by existing time marks. They are clustered in 2, 3 and 4 classes, and the number of classes with the lower entropy is selected. New time marks are obtained. This step is repeated until no acoustic segments larger than $60 \mathrm{~ms}$ remains.

2) Association of acoustic segments with phonetic units. A DTW algorithm which takes into account the following acoustic-phonetic rules is used to associate acoustic segments with phonetic units:

Rule (a). The association of an acoustic segment with a silence is penalized proportionally to the value of $E$. The association of an acoustic segment with a fricative or stop plosive phoneme is penalized with low values of $E$ and high values of $C C 1$. Finally, the association of an acoustic segment with other phonemes is penalized with low values of $E$ and with low values of $C C 1$.

Rule (b). The length of one or more consecutive acoustic segments associated with a phoneme is used to penalize the association if it is too short, except for stop plosive consonants and silences.

Rule (c). The length of an acoustic segment associated with a stop plosive consonant is used to penalize the association if it is larger than $30 \mathrm{~ms}$.

\section{3) Association of segments with phonetic units using phonetic prob-} abilities. The output of the previous association of acoustic segments with phonetic units is a primary segmentation used to estimate a GMM with several Gaussians per unit, typically 16. A DTW algorithm is also followed, but rule (a) is substituted by the use of phonetic probabilities from the GMM. Rules (b) and (c) are also used here with the same purpose. A new segmentation is 
obtained and a new GMM is estimated. This step is repeated until no changes in associations are found.

4) Forced alignment of phonetic units using phonetic probabilities. We follow the same strategy as before, but association of phonetic units with acoustic frames is not restricted by time marks. Rules (b) and (c) continue being applied. Actually, this step does the same alignment described in next subsection, but the phonetic probabilities used here are not as precise as the ones used in the progressive refinement.

\subsection{Progressive Refinement}

This is the core of our segmentation technique: Acoustical Clustering-Dynamic Time Warping (AC-DTW). It is based on unsupervised learning of acoustic classes and its association to phonemes by means of conditional probabilities. Each acoustic class represents a particular kind of acoustical manifestation and is modelled by a Gaussian distribution.

Phonetic boundaries are established by a DTW algorithm that uses the $a$ posteriori probability of each phonetic unit given an acoustic frame. These $a$ posteriori probabilities of phonemes are calculated by combining probabilities of acoustic classes, which are obtained from a clustering procedure on the acoustic feature space, and the conditional probabilities of each acoustic class with respect to each phonetic unit [5].

In the clustering procedure, it is assumed that acoustic classes can be modelled by means of Gaussian distributions. Parameters of each Gaussian distribution are estimated by using the unsupervised version of the Maximum Likelihood Estimation (MLE) procedure [6]. Thus, it is possible to estimate $\operatorname{Pr}\left(a \mid x_{t}\right)$, that is, the probability of each acoustic class $a$ from the set $A$ of acoustic classes, given an acoustic frame $x$ at time $t, x_{t}$, from the GMM. Nevertheless, as we need the probability of each phonetic unit $u$ from the set $U$ of phonetic units, given an acoustic vector $x_{t}, \operatorname{Pr}\left(u \mid x_{t}\right)$, a set of conditional probabilities are estimated in order to calculate the phonetic probabilities from the acoustic ones.

The use of conditional probabilities allows us to compute the phoneticconditional probability density $p\left(x_{t} \mid u\right)$ as follows [5]:

$$
p\left(x_{t} \mid u\right)=\sum_{a \in A} p\left(x_{t} \mid a\right) \cdot \operatorname{Pr}(a \mid u)
$$

for each $u \in U$, where $p\left(x_{t} \mid a\right)$ is the acoustic class-conditional probability density, computed as a Gaussian probability density function, and $\operatorname{Pr}(a \mid u)$ is the conditional probability that acoustic class $a$ has been manifested when phonetic unit $u$ has been uttered. Then, applying the Bayes formulation, we obtain the phonetic probabilities as:

$$
\operatorname{Pr}\left(u \mid x_{t}\right)=\frac{\sum_{a \in A} p\left(x_{t} \mid a\right) \cdot \operatorname{Pr}(a \mid u)}{\sum_{v \in U}\left(\sum_{a \in A} p\left(x_{t} \mid a\right) \cdot \operatorname{Pr}(a \mid v)\right)}
$$


for each $u \in U$. The DTW algorithm uses these a posteriori phonetic probabilities to align the frame sequence with the phonetic transcription.

The set of conditional probabilities $\operatorname{Pr}(a \mid u)$ for all $a \in A$ is initially computed from the coarse segmentation described in previous subsection. An iterative process updates the conditional probabilities until no improvements on segmentation are found.

\subsection{Boundary Adjustment}

A boundary adjustment is made from the segmentation previously obtained. This adjustment takes into account the values of several acoustic parameters to move phonetic boundaries. The parameters used at this stage are $d E$, the absolute value of first time derivative of Energy, $d Z$, the absolute value of first time derivative of zero crossing rate $(Z)$, and $d E d Z=d E * d Z$. Energy and $Z$ are computed every $2 \mathrm{~ms}$ using a $10 \mathrm{~ms}$ window.

Each phoneme boundary is adjusted using the gravity center formula with respect to a function inside a window centered in it. Both the function used and the window length depend on which phonetic units are related with the boundary:

- A stop plosive consonant followed by any other phoneme, gravity center of $d E$ calculated within a $20 \mathrm{~ms}$ window.

- A fricative consonant followed or preceded by any other phoneme, gravity center of $d E d Z$ calculated within a $60 \mathrm{~ms}$ window.

- Silence followed or preceded by any other phoneme, gravity center of $d E$ calculated within a $40 \mathrm{~ms}$ window.

- Vowel followed by other vowel, considered as a special case.

- Any other pair of consecutive phonetic units, gravity center of $d E$ plus $d E d Z$ calculated within a $40 \mathrm{~ms}$ window.

Boundaries between consecutive vowels are adjusted by dividing the sequence of frames from concatenating the two vowel segments into three subsegments with same length. Then it begins an iterative process which reduces the central segment as follows: if the first frame of the central segment is closer to the left segment than the central one, then that frame belongs to the left segment; by the other hand, if the last frame of the central segment is closer to the right segment than the central one, then that frame belongs to the right segment. When the central segment disappears or becomes unchanged, loop ends. In this last case, the adjusted boundary is fixed as the mean of the central segment boundaries.

\section{Segmentation Evaluation}

The evaluation criteria most widely used in the literature is to measure agreement of the obtained segmentation with respect to a manual segmentation. Usually the percentage of boundaries whose error is within a tolerance is calculated for a range of tolerances $1 / 27$. 
As discussed in the introduction, some researchers have wondered whether or not a manual segmentation is a valid reference [12. To evaluate it, they gave the same speech database to different human experts to segment it, and they evaluated the difference between them. In the study presented in [1], $97 \%$ of the boundaries within a tolerance of $20 \mathrm{~ms}$ were found and in 22 $93 \%$. We interpret this agreement as the maximum accuracy for a segmentation system, since a system that reaches $100 \%$ compared with a manual segmentation will at least differ around $95 \%$ with another manual segmentation for the same speech database.

\section{Experimental Results}

\subsection{Corpora}

In order to carry out experiments for both Spanish and English, we used two speech databases: Albayzin [8] and TIMIT [9], respectively.

The phonetic subcorpus from Albayzin database was used for the Spanish experiments: 6,800 utterances (around six hours of speech) obtained by making groups from a set of 700 distinct sentences uttered by 40 different speakers. 1,200 sentences manually segmented and labelled were used for testing, the remaining 5,600 sentences were used for training. No intersection speakers between training and testing subcorpora exist.

The TIMIT database was used for the English experiments: 6,300 utterances (approximately five hours of speech) by making groups of 10 sentences spoken by 630 speakers from 8 different dialect divisions of the United States. Two sentences were uttered by all speakers, the other eight sentences were selected from two phonetically rich sets. We used the suggested training/test subdivision 9 .

The same acoustic parameters were used on both databases. Each acoustic frame was formed by a 39-dimensional vector composed by the normalized energy, the first $12 \mathrm{Mel}$ frequency cepstral coefficients, and their first and second time derivatives. An acoustic frame is obtained using a $20 \mathrm{~ms}$ Hamming window at two different subsampling rates: $100 \mathrm{~Hz}$ (one frame every $10 \mathrm{~ms}$ ) and $200 \mathrm{~Hz}$ (one frame every $5 \mathrm{~ms}$ ) in order to study the influence of subsampling rate on segmentation accuracy.

\subsection{Coarse Segmentation}

As explained in subsection 2.1, the coarse segmentation is done in several steps. First, time marks are fixed where relevant acoustic changes are detected by means of statistical analysis of some acoustic parameters. Following, time marks are used to define acoustic segments which must be associated with phonemes in the phonetic transcription of each sentence. This association is refined until no changes are found. Then, the alignment of the frame sequence with the phonetic transcription begins. This alignment also repeats until no boundaries changes are detected. Table 1 shows the percentage of correctly fixed phonetic boundaries for the coarse segmentation. A set of tolerance intervals are considered. 
Table 1. Coarse segmentation. Percentage of correct phonetic boundaries within a set of tolerance intervals for the coarse segmentation.

\begin{tabular}{|l|c|c|c|c|c|}
\hline Database & $<5 \mathrm{~ms}$ & $<10 \mathrm{~ms}$ & $<15 \mathrm{~ms}$ & $<20 \mathrm{~ms}$ & $<30 \mathrm{~ms}$ \\
\hline \hline Albayzin & $35.7 \%$ & $59.0 \%$ & $70.0 \%$ & $76.2 \%$ & $83.3 \%$ \\
\hline TIMIT & $37.5 \%$ & $61.4 \%$ & $71.8 \%$ & $77.0 \%$ & $83.8 \%$ \\
\hline
\end{tabular}

\subsection{Boundary Adjustment}

The progressive refinement estimates a set of conditionals probabilities in an iterative process. The conditional probabilities are combined with the acoustical ones to obtain the a posteriori phonetic probabilities (see subsection 2.2). These phonetic probabilities are used by a DTW algorithm to align the acoustic frame sequence with the phonetic transcription. A phoneme segmentation is obtained as output, then a final boundary adjustment is done in order to improve the location of phoneme boundaries with respect to speech signal (see subsection 2.3).

Table 2 shows the percentage of correctly fixed phonetic boundaries obtained before and after applying the boundary adjustment when $100 \mathrm{~Hz}$ subsampling rate was used. Table 3 shows the same results when $200 \mathrm{~Hz}$ subsampling rate was used. It can be observed a significant improvement when the boundary adjustment is applied, specially for shorter tolerance intervals. In contrast, improvement when using higher subsampling rates is not as significant, even, there is no appreciable differences when using Albayzin database.

Another aspect to point out is the difference between both databases. This difference could reveal that our system is biased in favour of Spanish language. However, our results working on TIMIT database are similar to the ones reported in 7]. Their segmentation accuracy within a tolerance interval of $20 \mathrm{~ms}$ is $83.6 \%$, our segmentation accuracy is $84.7 \%$.

We made additional experiments using a set of manually segmented and labelled sentences as a starting point to the refinement process. Thus, we calculate the a posteriori probabilities using the best conditional probabilities we can obtain. Table 4 shows these segmentation results, which represent an upper bound of our segmentation technique, confirming that our system can learn without manually segmented and labelled sentences.

Table 2. Percentage of correct boundaries within a set of tolerances before and after the boundary adjustment. Subsampling rate $100 \mathrm{~Hz}$.

\begin{tabular}{|l|c|c|c|c|c|}
\hline Database & $<5 \mathrm{~ms}$ & $<10 \mathrm{~ms}$ & $<15 \mathrm{~ms}$ & $<20 \mathrm{~ms}$ & $<30 \mathrm{~ms}$ \\
\hline \hline Albayzin (before) & $41.0 \%$ & $67.2 \%$ & $80.4 \%$ & $87.3 \%$ & $94.0 \%$ \\
Albayzin (after) & $47.0 \%$ & $72.0 \%$ & $83.0 \%$ & $\mathbf{8 8 . 8} \%$ & $94.3 \%$ \\
\hline TIMIT (before) & $35.3 \%$ & $60.5 \%$ & $74.4 \%$ & $81.6 \%$ & $90.1 \%$ \\
TIMIT (after) & $40.6 \%$ & $65.9 \%$ & $77.1 \%$ & $82.9 \%$ & $89.9 \%$ \\
\hline
\end{tabular}


Table 3. Percentage of correct boundaries within a set of tolerances before and after the boundary adjustment. Subsampling rate $200 \mathrm{~Hz}$.

\begin{tabular}{|l|c|c|c|c|c|}
\hline Database & $<5 \mathrm{~ms}$ & $<10 \mathrm{~ms}$ & $<15 \mathrm{~ms}$ & $<20 \mathrm{~ms}$ & $<30 \mathrm{~ms}$ \\
\hline \hline Albayzin (before) & $40.5 \%$ & $65.5 \%$ & $79.7 \%$ & $87.6 \%$ & $94.2 \%$ \\
Albayzin (after) & $46.2 \%$ & $71.2 \%$ & $82.8 \%$ & $88.5 \%$ & $94.2 \%$ \\
\hline TIMIT (before) & $37.9 \%$ & $62.7 \%$ & $76.0 \%$ & $83.3 \%$ & $91.6 \%$ \\
TIMIT (after) & $42.4 \%$ & $67.9 \%$ & $79.0 \%$ & $\mathbf{8 4 . 7} \%$ & $91.2 \%$ \\
\hline
\end{tabular}

Table 4. Percentage of correct boundaries within a set of tolerances before and after the boundary adjustment when manually segmented and labelled sentences were used to estimate the conditional probabilities. Subsampling rate $200 \mathrm{~Hz}$.

\begin{tabular}{|l|c|c|c|c|c|}
\hline Database & $<5 \mathrm{~ms}$ & $<10 \mathrm{~ms}$ & $<15 \mathrm{~ms}$ & $<20 \mathrm{~ms}$ & $<30 \mathrm{~ms}$ \\
\hline \hline Albayzin (before) & $44.9 \%$ & $70.5 \%$ & $83.5 \%$ & $89.8 \%$ & $95.4 \%$ \\
Albayzin (after) & $50.2 \%$ & $74.8 \%$ & $85.6 \%$ & $90.7 \%$ & $95.5 \%$ \\
\hline TIMIT (before) & $41.1 \%$ & $65.9 \%$ & $79.3 \%$ & $85.9 \%$ & $92.9 \%$ \\
TIMIT (after) & $46.1 \%$ & $71.2 \%$ & $81.7 \%$ & $86.8 \%$ & $92.6 \%$ \\
\hline
\end{tabular}

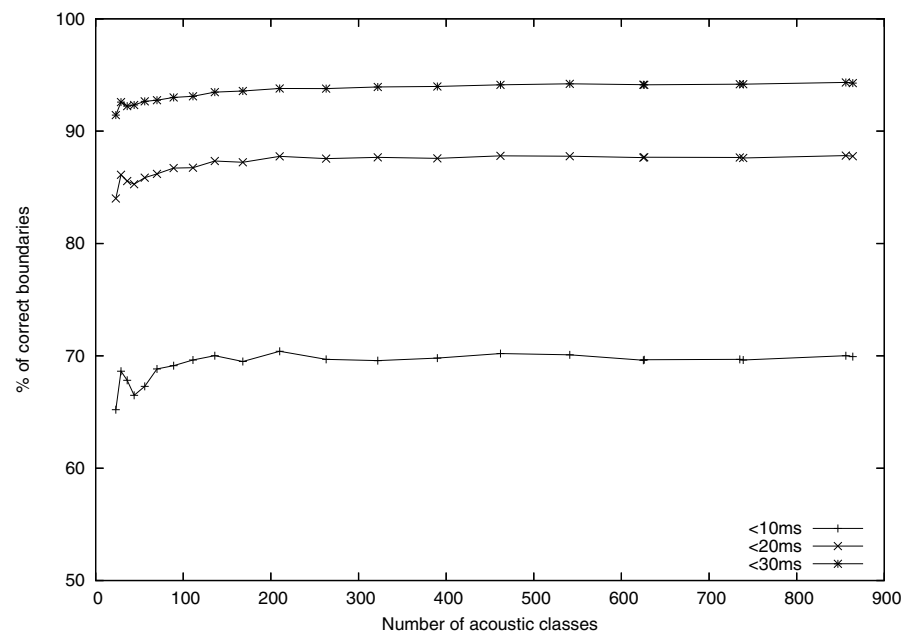

Fig. 1. Percentage of correct phonetic boundaries versus the number of acoustic classes using Albayzin database and $100 \mathrm{~Hz}$ subsampling rate. Tolerance intervals of 10,20 and $30 \mathrm{~ms}$ are presented.

In order to study the influence of the number of acoustic classes an exploratory experiment was performed. The progressive refinement, explained in Section 2.2. was repeated for a set of GMM with different number of mixture components. Each GMM with a particular number of Gaussian distributions is the product of each intermediate step in the hiearchical clustering procedure applied to 
estimate the "natural" acoustic classes. Figure 1 shows the segmentation accuracy obtained for different values of the number of acoustic classes. There is no significant improvement from 200 acoustic classes.

\section{Conclusions}

In this work, we have presented a fully automatic system to segment speech databases without the need for a manually segmented subset. This task is important in order to obtain segmented databases for training phoneme-based speech recognizers or selecting phonetic units in TTS systems.

The improvement in coarse segmentation stage has impact in the final segmentation. The results obtained using the conditional probabilities estimated from a set of manually segmented and labelled sentences represent an upper bound of our technique. The small difference with respect to the automatic system validates our technique, which no uses manually segmented and labelled sentences at all. The segmentation accuracy obtained here for TIMIT database is similar to the results presented in other works with the same database using more complex methods for the final adjustment.

\section{References}

1. Toledano, D.T., Hernández Gómez, L., Villarrubia Grande, L.: Automatic Phonetic Segmentation. IEEE Transactions on Speech and Audio Processing 11(6), 617-625 (2003)

2. Kipp, A., Wesenick, M.B., Schiel, F.: Pronunciation modelling applied to automatic segmentation of spontaneous speech. In: Proceedings of Eurospeech, Rhodes, Greece, pp. 2013-2026 (1997)

3. Adell, J., Bonafonte, A., Gómez, J.A., Castro, M.J.: Comparative study of automatic phone segmentation methods for TTS. In: IEEE ICASSP, Philadelphia, USA, vol. 1, pp. 309-312 (2005)

4. Pikrakis, A., Giannakipoulos, T., Theodoridis, S.: A Speech/Music Discriminator of Radio Recordings Based on Dynamic Programming and Bayesian Networs. IEEE Trans. on Multimedia 10, 846-857 (2008)

5. Gómez, J.A., Castro, M.J.: Automatic Segmentation of Speech at the Phonetic Level. In: Caelli, T.M., Amin, A., Duin, R.P.W., Kamel, M.S., de Ridder, D. (eds.) SPR 2002 and SSPR 2002. LNCS, vol. 2396, pp. 672-680. Springer, Heidelberg (2002)

6. Duda, R.O., Hart, P.E., Stork, D.G.: Pattern Classification, 2nd edn. John Wiley \& Sons, Chichester (2001)

7. Mporas, I., Ganchev, T., Fakotakis, N.: A Hybrid Architecture for Automatic Segmentation of Speech Waveforms. In: IEEE ICASSP 2008, Las Vegas, USA, pp. 4457-4460 (2008)

8. Moreno, A., Poch, D., Bonafonte, A., Lleida, E., Llisterri, J., Mariño, J.B., Nadeu, C.: Albayzin Speech Database: Design of the Phonetic Corpus. In: Eurospeech 1993, Berlin, Germany, September 1993, vol. 1, pp. 653-656 (1993)

9. TIMIT Acoustic-Phonetic Continuous Speech Corpus, National Institute of Standards and Technology Speech Disc 1-1.1, NTIS Order No. PB91-5050651996 (October 1990) 